\title{
Effects of hydrodynamic heterogeneity on mineral reactivity and nucleation in porous and fractured porous media
}

\author{
JENNA POONOOSAMY ${ }^{1}$, SOPHIE ROMAN ${ }^{2}$, CYPRIEN \\ SOULAINE $^{2}$, YUANKAI YANG ${ }^{1}$, DIRK BOSBACH ${ }^{1}$ AND \\ GUIDO DEISSMANN ${ }^{1}$
}

${ }^{1}$ Forschungszentrum Jülich GmbH

${ }^{2}$ Earth Sciences Institute of Orleans, Univ. Orleans, CNRS, BRGM

Presenting Author: g.deissmann@fz-juelich.de

Fluid/rock interactions leading to precipitation and dissolution of minerals in porous or fractured porous media are important processes governing the evolution of many subsurface systems, such as oil and gas reservoirs, geothermal systems, or engineered systems for $\mathrm{CO}_{2}$ sequestration or nuclear waste disposal. These processes lead to modifications of pore architecture, porosity/permeability, and other physical characteristics of the rock matrix that can have significant effects on subsurface solute transport. The early stage of mineral precipitation, i.e., the nucleation process, strongly affects the pore network alteration and consequent changes in transport properties, since nucleation in the pore space (homogeneous nucleation) or nucleation on the surface of minerals (heterogeneous nucleation) will alter the hydraulic pathways of porous media differently. While the former can have considerable effects on porosity, the latter is more likely to cause a reduction of the size of pore throats. The implementation of such coupled processes into (continuumscale) numerical reactive transport codes requires a mechanistic process understanding on the pore-scale and the development of adequate methods for upscaling.

In this context, we developed a new generation of flowthrough microfluidic reactors coupled with high-resolution Raman imaging that enables in situ and non-destructive 4D assessment of mineral reactivity with full spatio-temporal resolution of the chemical and porosity evolution of the system at the pore-scale. Our investigations using complementary porescale modelling to map the velocity fields enabled a systemic evaluation of the impact of the Peclet number $\left(\mathrm{P}_{\mathrm{e}}\right)$ and the hydrodynamic heterogeneity on nucleation mechanisms in porous and fractured porous media. Although it is typically expected that homogeneous and heterogeneous nucleation occur at high and low saturation state, respectively, our study showed that homogeneous nucleation can also take place at low saturation state, provided that the flow velocities are high enough such that the mixing of the solutes will enable the formation of nucleation clusters. 